Journal of Nuclear and Radiochemical Sciences, Vol. 4, No. 1, pp. 5-7, 2003

\title{
Half-Life of Samarium-147
}

\author{
Norikazu Kinoshita, ${ }^{*, a}$ Akihiko Yokoyama, ${ }^{\text {b }}$ and Takashi Nakanishi ${ }^{\mathbf{b}}$ \\ ${ }^{\mathrm{a}}$ Graduate School of Natural Science and Technology, Kanazawa University, Kanazawa 920-1192, Japan \\ ${ }^{\mathrm{b}}$ Department of Chemistry, Faculty of Science, Kanazawa University, Kanazawa 920-1192, Japan
}

Received: April 16, 2003; In Final Form: June 2, 2003

The alpha-decay half-life of ${ }^{147} \mathrm{Sm}$ has been reevaluated. Known amounts of natural Sm and an alpha-emitter standard $\left({ }^{210} \mathrm{Po},{ }^{238} \mathrm{U}\right.$, or $\left.{ }^{241} \mathrm{Am}\right)$ were mixed well to prepare thin sources for the simultaneous counting of ${ }^{147} \mathrm{Sm}$ and the alpha-emitter standard by means of an alpha-spectrometer using a silicon surface barrier detector. The alpha-disintegration rate of known amounts of ${ }^{147} \mathrm{Sm}$ was determined by reference to the alpha activity of the standard. The source preparation and counting were repeated to establish the reproducibility of the present half-life determination, and supplementary alpha spectrometry was carried out by a liquid-scintillation spectrometer. The arithmetic mean of the experimental half-life values was obtained to be $(1.17 \pm 0.02) \times 10^{11} \mathrm{y}$. This value is about $10 \%$ longer than the currently adopted value, $(1.06 \pm 0.02) \times 10^{11} \mathrm{y}$, and the possible factors for this difference are discussed.

\section{Introduction}

Samarium-147 is a naturally occurring long-lived alpha emitter (natural isotopic abundance: $14.99 \%$; currently adopted half-life: $1.06 \times 10^{11} \mathrm{y} ;{ }^{1}$ alpha-ray energy: $2.235 \mathrm{MeV}$ ). The nuclide has recently been proved to be useful as an internal reference in the determination of alpha activity of $\mathrm{mBq}$ level by alpha-spectrometric technique named $\mathrm{Sm}$-method. ${ }^{2-5}$

The present authors are searching for ${ }^{244} \mathrm{Pu}$ and ${ }^{146} \mathrm{Sm}$, now extinct in the solar system, in deep-sea sediment to obtain evidence for injection of materials from a hypothetical nearEarth supernova. ${ }^{6,7}$ In the research project searching for the extinct nuclides in deep-sea sediment, it has been planned to reevaluate the half-life of ${ }^{146} \mathrm{Sm}$ (an alpha emitter; currently adopted half-life: $1.03 \times 10^{8} \mathrm{y}{ }^{8}$ alpha-ray energy: $2.455 \mathrm{MeV}$ ) by reference to the half-life of ${ }^{147} \mathrm{Sm}$, because there is large scatter in the literature values on the half-life of ${ }^{146} \mathrm{Sm} .{ }^{8-10}$ The currently adopted half-life of ${ }^{147} \mathrm{Sm}$ was determined in 1970 to be $(1.06 \pm 0.02) \times 10^{11} \mathrm{y},{ }^{1}$ whereas the half-life values reported from 1954 to 1970 showed some scatter between $1.04 \times 10^{11} \mathrm{y}$ and $1.28 \times 10^{11} \mathrm{y}^{1,11-15}$ Hence, it is worthwhile to reevaluate the half-life of ${ }^{147} \mathrm{Sm}$ by means of a developed alpha-spectrometer with the counting sources prepared in a manner different from those adopted in the earlier works.

\section{Experimental}

Materials. Each of two kinds of commercially available $\mathrm{Sm}_{2} \mathrm{O}_{3}$ reagents of $99.9 \%$ purity supplied by two makers was dissolved in conc. $\mathrm{HNO}_{3}$ followed by dilution with distilled water to prepare known concentration (in $\mu \mathrm{g}-\mathrm{Sm} / \mathrm{g}$-solution) of two kinds of Sm standard solutions (hereinafter these are described as Sm-A and Sm-B). In addition, two kinds of commercially available $\mathrm{Sm}$ standard solutions for atomic absorption spectrometry supplied by two makers were also used (hereinafter these are described as Sm-C and Sm-D). Radioactive and non-radioactive impurities in the $\mathrm{Sm}$ solutions were measured by low-level gamma spectrometry, neutron activation method, ICP-MS spectrometry, and thermal ionization mass spectrometry, and it was confirmed that the solutions

*Corresponding author. E-mail: n-kino@stu.kanazawa-u.ac.jp. FAX: +81-76-264-5742. did not contain any detectable impurities and that the isotopic composition is natural.

Calibrated solutions of alpha-radioactive nuclide were used as internal standards in preparation of counting sources for alpha spectrometry: ${ }^{210} \mathrm{~Pb}-{ }^{210} \mathrm{Po}$ supplied by LMRI, France, natural uranium supplied by NBS, U.S.A., and ${ }^{241} \mathrm{Am}$ supplied by CERCA, France.

Apparatus. Alpha-spectrometry was carried out using an EG \& G Ortec Octet ${ }^{\mathrm{TM}} \mathrm{PC}$ alpha-spectrometer equipped with a silicon surface-barrier detector of $450 \mathrm{~mm}^{2}$ for active area. A counting source was placed in the vacuum $(<4 \mathrm{~Pa})$ chamber of the spectrometer with a solid angle of $\sim 20 \%$ of $4 \pi$. Typical energy resolution of the alpha-spectrometer was $\sim 50 \mathrm{keV}$ (FWHM) for alpha-particles from ${ }^{147} \mathrm{Sm}$, and typical background of the spectrometer was $2 \sim 5 \mathrm{cpd}$ in each peak region of ${ }^{147} \mathrm{Sm}(2.235 \mathrm{MeV}),{ }^{210} \mathrm{Po}(5.034 \mathrm{MeV}),{ }^{238} \mathrm{U}(4.198 \mathrm{MeV})$, and ${ }^{241} \mathrm{Am}(5.486 \mathrm{MeV})$.

For a supplementary alpha-spectrometry of the mixture of Sm and ${ }^{241} \mathrm{Am}$, liquid-scintillation spectrometry was carried out using an Aloka LSC-6100 liquid-scintillation spectrometer.

Procedure. Known aliquot (weight basis) of Sm-A, Sm-B, $\mathrm{Sm}-\mathrm{C}$, or Sm-D solution was mixed well with known amount (weight basis) of calibrated ${ }^{210} \mathrm{Po}$, natural uranium, or ${ }^{241} \mathrm{Am}$ solution. From the four Sm solutions and the three alphaemitter standards, 10 kinds of combinations of the Sm solutions and the alpha-emitter standards were made. The mixture was then evaporated on a watch glass $(30 \mathrm{~mm} \phi)$ to prepare thin deposit for alpha-spectrometry. The amount of Sm mounted on the watch glass was adjusted to $\sim 100 \mu \mathrm{g}$ (i.e., $\sim 0.012 \mathrm{~Bq}$ of ${ }^{147} \mathrm{Sm}$ ), and the amount of the alpha-emitter standard was adjusted to $\sim 0.04 \mathrm{~Bq}$.

Alpha spectrometry using a silicon surface-barrier detector was carried out for 1 to 2 weeks for each of the counting sources prepared by the method described above, and alphadisintegration rate of the known amount of ${ }^{147} \mathrm{Sm}$ was determined by reference to the alpha-activity of internal standard $\left({ }^{210} \mathrm{Po},{ }^{238} \mathrm{U}\right.$, or $\left.{ }^{241} \mathrm{Am}\right)$ (Figure 1).

The measurement of alpha-disintegration rate of the known amount of ${ }^{147} \mathrm{Sm}$ was also carried out concurrently by means of a liquid-scintillation (LS) spectrometer. For the LS spectrometry only ${ }^{241} \mathrm{Am}$ was used as the internal standard: known amounts of the Sm standard solution and ${ }^{241} \mathrm{Am}$ standard solution were taken in an LS vial. The solution in the vial was 


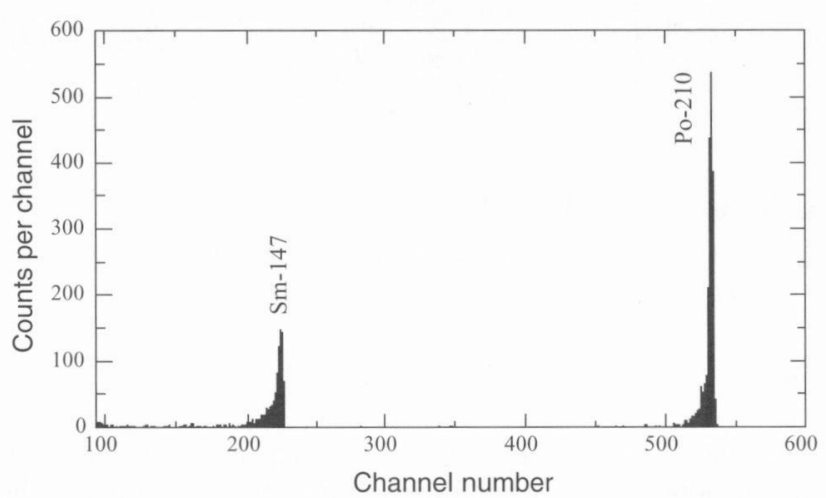

Figure 1. An example of alpha-spectra obtained with alpha-spectrometer using silicon surface barrier detector. This spectrum was obtained after $\sim 6.6$ days counting for a counting source prepared from the mixture of $\sim 100 \mu \mathrm{g}$ of Sm and $\sim 0.04 \mathrm{~Bq}$ of ${ }^{210} \mathrm{Po}$.

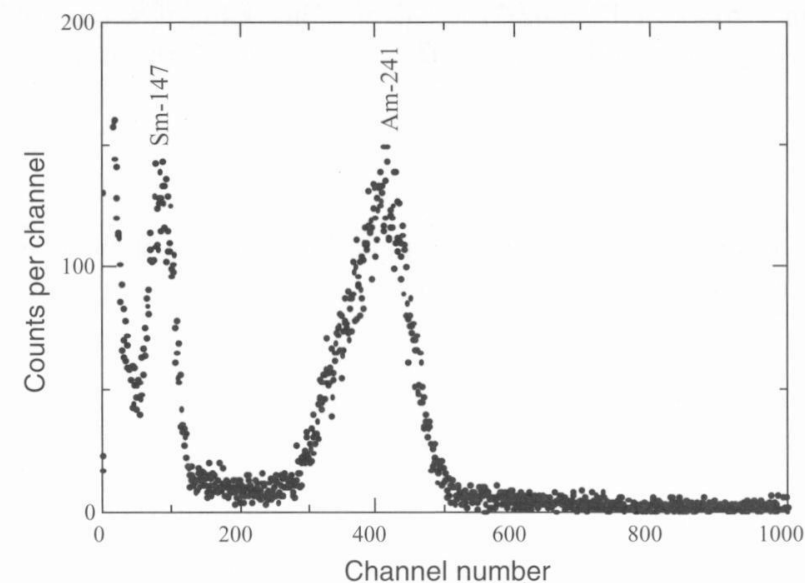

Figure 2. An example of alpha-spectra obtained with liquid-scintillation spectrometer. This spectrum was obtained after 5 hours counting for a mixture of $\sim 2 \mathrm{mg}$ of $\mathrm{Sm}$ and $\sim 0.8 \mathrm{~Bq}$ of ${ }^{241} \mathrm{Am}$ dissolved in the Amersham ACS II scintillation cocktail.

evaporated to dryness on a hot plate $\left(\sim 200{ }^{\circ} \mathrm{C}\right)$, and addition of $\sim 1 \mathrm{~mL}$ of $\mathrm{HCl}$ followed by evaporation to dryness was repeated three times. To dissolve $\mathrm{Sm}$ and $\mathrm{Am}, 1 \mathrm{~mL}$ of glacial $\mathrm{CH}_{3} \mathrm{COOH}$ and $1 \mathrm{~mL}$ of 2-ethylhexanoic acid were successively added to the vial. The content in the vial was then heated on a hot plate $\left(200{ }^{\circ} \mathrm{C}\right)$ to evaporate only $\mathrm{CH}_{3} \mathrm{COOH}$. Finally, $10 \mathrm{~mL}$ of the Amersham ACS II scintillation cocktail (or p-terphenyl + POPOP + toluene scintillation cocktail) was added. The LS counting was continued for 5 hours for each of the counting sources (Figure 2). A blank sample for background LS measurement was also prepared in the same way as mentioned above.

Calculation of the Half-Life Value. For the calculation of the half-life value of ${ }^{147} \mathrm{Sm}$, it was assumed that $\mathrm{Sm}$ and the standard alpha-emitting nuclide were not fractionated throughout the present procedure to prepare the counting sources.

Based on the data obtained by alpha-spectrometry, alphaactivity of ${ }^{147} \mathrm{Sm}$ in the counting source was determined by reference to the alpha-activity of the internal standard in the same counting source:

$$
A_{147}=\frac{C_{147}}{C_{\mathrm{STD}}} \times A_{\mathrm{STD}}
$$

where $A_{147}$ is the alpha-activity of ${ }^{147} \mathrm{Sm}, A_{\mathrm{STD}}$ is the alphaactivity of the alpha-emitter standard, $C_{147} / C_{\mathrm{STD}}$ is the ratio of the background subtracted peak area of ${ }^{147} \mathrm{Sm}$ to that of the alpha-emitter standard. On the other hand, the alpha-activity of ${ }^{147} \mathrm{Sm}$ is expressed as:

$$
A_{147}=\frac{\ln 2}{T_{147}} \times N_{147}
$$

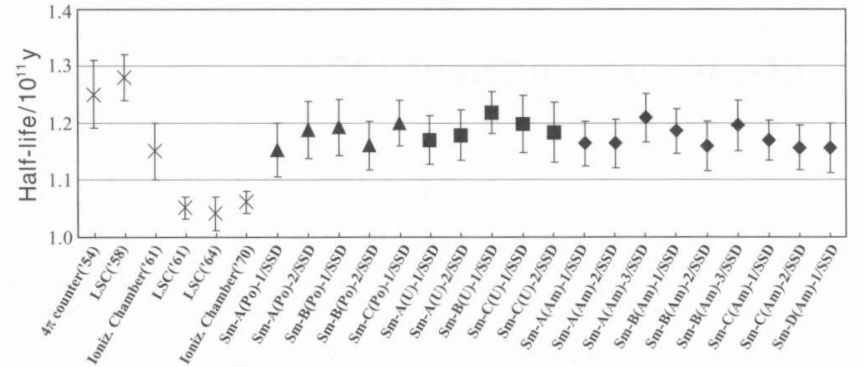

Figure 3. Half-life values and their errors determined with alphaspectrometer using silicon surface barrier detector. The literature values are indicated by cross symbols, and values obtained in this work are indicated by closed symbols. The prepared sources are referred to as the sample names depicted in the figure with the original solution name, the reference standard, source number, and the measurement method.

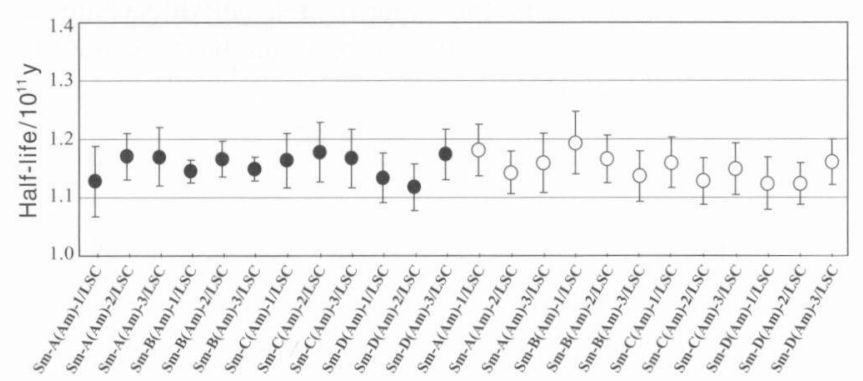

Figure 4. Half-life values determined with liquid-scintillation spectrometer. The prepared sources are depicted as same as Figure 3. Amersham ACS II scintillation cocktail (closed symbol) and pterphenyl + POPOP + toluene scintillation cocktail (open symbol) were used.

where $T_{147}$ is half-life of ${ }^{147} \mathrm{Sm}$ and $N_{147}$ is the number of ${ }^{147} \mathrm{Sm}$ atoms in the counting source. Equation 3 is drawn from eq 1 and eq 2 .

$$
T_{147}=\frac{N_{147} \times \ln 2}{\frac{C_{147}}{C_{\mathrm{STD}}} \times A_{\mathrm{STD}}}
$$

The value of $N_{147}$ can be calculated from the concentration of $\mathrm{Sm}$ in the Sm-standard solution, weight of Sm standard solution taken for the preparation of the counting source, Avogadro constant, isotopic abundance of ${ }^{147} \mathrm{Sm}$ in natural $\mathrm{Sm}$, and atomic weight of Sm. Since it can be assumed that $\mathrm{Sm}$ and alpha-emitter standard do not fractionate throughout the present procedure to prepare counting source, $N_{147} / A_{\mathrm{STD}}$ ratio is virtually invariant. The half-life of ${ }^{147} \mathrm{Sm}\left(T_{147}\right)$ can be calculated by substituting the experimental data of $N_{147} / A_{\mathrm{STD}}$ and $C_{147} / C_{\mathrm{STD}}$ for eq 3 .

\section{Results and Discussion}

A series of half-life values of ${ }^{147} \mathrm{Sm}$ measured by means of an alpha-spectrometer using a silicon surface barrier detector are shown in Figure 3 together with literature values. ${ }^{11-15} \mathrm{~A}$ series of half-life values of ${ }^{147} \mathrm{Sm}$ measured with an LS spectrometer are shown in Figure 4. The attached error for each half-life includes nominal systematic errors in the calibration of the alpha-emitter standard and in the measurement of isotopic abundance of ${ }^{147} \mathrm{Sm}$ in addition to the statistical error of $1 \sigma$ in alpha counting. The values shown in Figures 3 and 4 agree very closely with each other within the error.

In alpha-spectrometry for a deposited counting source, selfabsorption of alpha-rays in the source is a serious problem; energy loss and absorption of alpha-particles in the windowlayer of silicon surface barrier detector $(\sim 50 \mathrm{~nm})$ and in vacuum chamber $(<4 \mathrm{~Pa})$ are negligible. As to the extent of self-absorption in the present alpha-spectrometry, since the 
thickness of the residue of Sm and the alpha-emitter standard on the watch glass is less than $15 \mu \mathrm{g} / \mathrm{cm}^{2}$, energy loss and absorption of alpha-particles from ${ }^{147} \mathrm{Sm}$ and the alpha-emitter standard of the counting source can be expected to be negligible.

The counting efficiency of the LS spectrometer is high $(\sim 100 \%)$, however, the spectrometer has disadvantages of high background and poor energy-resolution (Figure 2). Hence, in spite of the agreement between the mean value measured with the LS spectrometer, $(1.15 \pm 0.02) \times 10^{11} \mathrm{y}$, and the mean value with the surface barrier detector, $(1.17 \pm 0.02) \times 10^{11} \mathrm{y}$, within the error, the former value was regarded as a supporting data for the latter because of the disadvantages described above.

The arithmetic mean of the half-life values obtained in this work for the nineteen counting sources (Figure 3$)$ is $(1.17 \pm$ $0.02) \times 10^{11} y$. The error associated with the average value is one standard deviation. The average value is about $10 \%$ longer than the currently adopted value, $(1.06 \pm 0.02) \times 10^{11} \mathrm{y}$.

In the earlier works, the half-life of ${ }^{147} \mathrm{Sm}$ was measured with a $4 \pi$ gas-flow counter, ${ }^{11}$ LS spectrometers, ${ }^{12,14,15}$ and ionization chambers. ${ }^{1,13}$ According to the conventional method for the determination of long half-lives, the half-life value for ${ }^{147} \mathrm{Sm}\left(T_{147}\right)$ is deduced by substituting measured values of $A_{147}$ and $N_{147}$ (see eq 2 ) in the following equation:

$$
T_{147}=\frac{\ln 2}{A_{147}} \times N_{147} \text {. }
$$

Hence, experimental errors in measuring $A_{147}$ and $N_{147}$ give inaccurate results for $T_{147}$. Impurities in $\mathrm{Sm}$ reagent bring error into the value of $N_{147}$, and uncertainty of counting efficiency, self-absorption of counting source and radioactive impurities in Sm reagent bring error into the value of $A_{147}$. It is first suspected that the purity of $\mathrm{Sm}$ reagent was not sufficient in the earlier works. It is also suspected that the corrections for counting efficiency and self-absorption were not appropriate in the earlier works. High background and poor energy resolution of LS spectrometers used in the earlier works might also result in inaccurate ${ }^{147} \mathrm{Sm}$ half-life.

In contrast to the earlier works, in the present cautious work, all the error sources mentioned above were excluded. Hence, we conclude that the result obtained in this work is reliable.
Acknowledgements. We would like to thank Dr. K. Norisuye of Institute for Chemical Research, Kyoto University for the thermal ionization mass spectrometry. We also wish to acknowledge the assistance of the members of the Research Reactor Institute, Kyoto University in the neutron activation analysis.

\section{References}

(1) M.C. Gupta and R.D. MacFarlane, J. Inorg. Nucl. Chem. 32, 3425 (1970).

(2) T. Mitsugashira, M. Hara, Y. Suzuki, M. Watanabe, and H. Hirayama, Proc. 1996 SERNIA Symp. on Environmental Radioactive Impact in Asia, 6-8 Sep. 1996, Taipei, ROC, p. 291 (1996).

(3) T. Mitsugashira, M. Hara, Y. Suzuki, and M. Watanabe, Res. Rept. LNS Tohoku Univ. 30, 47 (1997).

(4) T. Mitsugashira, M. Hara, Y. Suzuki, M. Watanabe, S. Hirai, Y. Okada, and A. Mori, J. Radioanal. Nucl. Chem. 239, 345 (1999).

(5) T. Mitsugashira, M. Hara, P. Kim, K. Nakashima, and K. Nakayama, J. Radioanal. Nucl. Chem. 255, 201 (2003).

(6) K. Sakamoto, Y. Hashimoto, and T. Nakanishi, in Origin of Elements in the Solar system: Implications of Post-1957 Observations, edited by O. Manuel (Kluwer Academic/ Plenum Publishers, New York, 2000), p. 511.

(7) M. Paul, A. Valenta, I. Ahmad, D. Berkovits, C. Bordeanu, S. Ghelberg, Y. Hashimoto, A. Hershkowitz, S. Jiang, T. Nakanishi, and K. Sakamoto, Astrophys. J. 558, L133 (2001).

(8) A.M. Friedman, J. Milsted, D. Metta, D. Henderson, J. Lerner, A. L. Harkness, and D.J. Rokop, Radiochim. Acta 5, 192 (1966).

(9) D.C. Dunlavey and G.T. Seaborg, Phys. Rev. 92, 206 (1953).

(10) M. Nurmia, G. Graeffe, K. Valli, and J. Aaltonen, Ann. Acad. Sci. Fenn. A VI, 148, 3 (1964).

(11) G. Beard and M.L. Wiedenbeck, Phys. Rev. 95, 1245 (1954).

(12) G.B. Beard and W.H. Kelly, Nucl. Phys. 8, 207 (1958).

(13) R.D. MacFarlane and T.P. Kohman, Phys. Rev. 121, 1758 (1961).

(14) P.M. Wright, E.P. Steinberg, and L.E. Glendenin, Phys. Rev. 123, 205 (1961).

(15)D. Donhoffer, Nucl. Phys. 50, 489 (1964). 